Pacific Journal of Mathematics

SEMI-GROUPS OF SCALAR TYPE OPERATORS IN BANACH 


\title{
SEMI-GROUPS OF SCALAR TYPE OPERATORS IN BANACH SPACES
}

\author{
T. V. PANChapagesan
}

This paper deals with the spectral representation theorems of semi-groups of scalar type operators in Banach spaces. These results generalize the corresponding ones on semi-groups of hermitian, normal and unitary operators in Hilbert spaces. In the beginning sections we study some interesting properties of a $W^{*}(\|\cdot\|)$-algebra-which generalizes the notion of an abelian von Neumann algebra to Banach spaces-and unbounded spectral operators arising out of $E(\cdot)$-unbounded measurable functions where $E(\cdot)$ is a resolution of the identity. These results are applied later to prove the spectral representation theorems on semi-groups of scalar type operators. The last theorem of this paper gives an extension of Stone's theorem on strongly continuous one parameter group of unitary operators to arbitrary Banach spaces.

This paper mainly deals with the spectral representation theorems of semi-groups of scalar type operators in Banach spaces, generalizing those of semi-groups of hermitian, normal and unitary operators in Hilbert spaces. Since all the classical proofs of these theorems vitally depend on the inner-product structure of the Hilbert space they cannot be adapted to Banach spaces. However, Phillips has obtained in [15] these spectral representation theorems on Hilbert spaces by making use of the theory of abelian $W^{*}$ (von Neumann) algebras. Here we adapt his method of proof by suitably generalizing the notion of an abelian $W^{*}$ algebra to Banach spaces.

In [3] Bade has developed the theory of operator algebras $W$ on Banach spaces, which are generated in the weak operator topology by a $\sigma$-complete Boolean algebra of projections. Such an algebra $W$ has its maximal ideal space extremally disconnected, just as in the case of an abelian $W^{*}$ algebra. However, $W$ is not a $B^{*}$-algebra. To this end we exploit the work on hermitian operators in Banach spaces by Berkson [4, 5, 6], Lumer [12,13] and Vidav [18] and we define an algebra called a $W^{*}(\|\cdot\|)$-algebra in $\S 2$ of this paper, which is a $B^{*}$-algebra generated in the weak operator topology by a $\sigma$-complete Boolean algebra of projections. The involution * of this algebra is also strongly continuous (Theorem 1 of $\S 2$ ) as its counterpart in the abelian $W^{*}$ algebra. Thus $W^{*}(\|\cdot\|)$-algebras have all the essential properties of abelian $W^{*}$ algebras, though the double commutant theorem fails for such algebras. (See Dieudonné [7]).

Further in $\S 2$ we introduce an ordering relation among hermitian 
operators on a Banach space and prove that in a $W^{*}(\|\cdot\|)$-algebra a bounded monotonic net of operators converges strongly. This result is given here not only for its own interest, but also for its application later in Lemma 6 of $\S 4$.

The spectral operators arising from unbounded measurable functions are studied in $\S 3$ and a generalization of Lemma 6 of Dunford [8] is obtained here in Theorem 4. This is a basic result, which is used in the theory of semi-groups of scalar type operators to show that the infinitesimal generator of the semi-group is a spectral operator of scalar type. Theorem 5 of this section, which states that the residual spectrum of an unbounded spectral operator of scalar type is empty, generalizes the corresponding result for maximal normal operators in Hilbert spaces.

In $\S \S 4,5$ and 6 we study the semi-groups of scalar type operators making use of the tools developed in $\S 2$ and $\S 3$. We generalize Theorems 22.3.1, 22.3.2, 22.4.1, 22.4.2 and 22.4.3 of Hille and Phillips [11] to Banach spaces. Because of the lack of the inner-product in our case the relations (13) and (23) are to be obtained here in a way completely different from [11]. Also Theorem 8 of $\S 4$ has to be weaker than the corresponding Theorem 22.3.2 of [11] as we have to apply here the generalized Lebesgue bounded convergence theorem which is available only for a sequence of functions.

As the $W^{*}(\|\cdot\|)$-algebra $W$ does not in general satisfy the double commutant theorem, we have to assume explicitly in Theorem 9 of $\S 5$, that the resolvent operators $R(\lambda, A)$ of the infinitesimal generator $A$ of the semi-group belong to $W$. However, this explicit assumption is not needed in the particular case when the operators of the semigroup are all unitary. (See Theorem 10 of $\S 6$ ).

1. Preliminaries. The terminology and notation in this paper are as follows. By a Banach space we mean a complex Banach space. $X$ always denotes a Banach space. For definitions of boundedness, $\sigma$-completeness and completeness of a Boolean algebra (abbreviated as B.A. hereafter) of projections in $X$, one may refer to Bade [3]. For a B.A. $\mathfrak{B}$ of projections in $X, \overline{\mathfrak{B}}^{s}$ denotes the strong closure of $\mathfrak{B}$. The results on spectral operators that are used in the sequel, can be found in Dunford [8] and Bade [1, 2, 3].

Definition 1. An element $h$ of a Banach algebra $A$ with the norm $\|\cdot\|$ will be called hermitian in the norm if for $r$ real,

$$
\|e+i r h\|=1+o(r)
$$

as $r \rightarrow 0$, where $e$ is the identity of $A$. 
If $T$ is an operator on a Banach space $X$ then $T$ is called hermitian in the equivalent norm $\|\cdot\|$ of $X$ in the sense of Vidav if $T$, as an element of the algebra $\mathfrak{B}(X)$, is hermitian in the induced operator norm $\|\cdot\|$ of $\mathfrak{B}(X)$.

Definition 2. An operator $T$ on a Banach space $X$ is said to be hermitian in the equivalent norm $\|\cdot\|$ of $X$ in the sense of Lumer, if $[T x, x]$ is real for all $x$ in $X$ with $\|x\|=1$ where [, ] is a semi-innerproduct (see Lumer [12]) on $X$ consistent with the norm $\|\cdot\|$.

In [12], Lumer has shown that $T$ is hermitian in $\|\cdot\|$ in the sense of Lumer if and only if $T$ is hermitian in the sense of Vidav. Hence we write that $T$ is hermitian in the norm $\|\cdot\|$ to mean hermiticity in either sense.

(1.1) VidaV's THEOREM. Let $A$ be a Banach algebra with the identity and with the norm $\|\cdot\|$. Let $H$ be the set of elements of $A$ which are hermitian in $\|\cdot\|$. If $A=H+i H$ and if for every $h \in H$, $h^{2}$ can be expressed in the form $h^{2}=u+i v$ with $u, v$ in $H$ and $u v=$ $v u$, then

(i) for each $x$ in $A$, the decomposition $x=u+i v u, v$ in $H$, is unique;

(ii) the map * which assigns to each element $x=u+i v$ (where $u, v$ are in $H$ ) the element $x^{*}=u-i v$ is an involution on $A$; (we call $x^{*}$ the Vidav adjoint of $x$ );

(iii) $\|\cdot\|_{0}$, defined by $\|x\|_{0}=\left\|x^{*} x\right\|^{1 / 2}$ is a Banach algebra norm on $A$ equivalent to the given norm and moreover $\|h\|_{0}=\|h\|$ for every $h \in H$;

(iv) the algebra $A$ with the involution * and the norm $\|\cdot\|_{0}$, is a $B^{*}$-algebra.

Definition 3. If a Banach algebra $A$ with the norm $\|\cdot\|$ satisfies the hypothesis of Vidav's theorem (1.1) and is equipped with the involution * defined in (ii) of (1.1), then we call $A$, following Berkson [5], a $V^{*}$-algebra in the norm $\|\cdot\|$.

The Vidav's theorem (1.1) has been sharpened recently by Berkson in [5] to the following form.

(1.2) $A$ is a $V^{*}$-algebra in the norm $\|\cdot\|$ if and only if $A$ is a $B^{*}$-algebra in the norm $\|\cdot\|$.

As pointed out to the author by Bade and Phillips, the above result of Berkson has the following important consequence for the 
theory of spectral operators.

Theorem. (1.3) Let $\mathfrak{B}$ be a bounded B.A. of projections on a Banach space $X$ and let $A$ be the uniformly closed Banach algebra generated by $\mathfrak{B}$. Then there exists an equivalent norm $\|\cdot\| \|$ on $X$ (i.e., a norm ||$\cdot|| \mid$ which is equivalent to the given norm of $X$ ) such that the norm of each operator in A, computed relative to $\|\cdot \cdot\|$, is equal to its spectral norm. Thus the Gelfand map of $A$ is an isometric isomorphism onto $C(\mathrm{~m}), \mathfrak{m}$ the space of maximal ideals of $A$.

Proof. Since $\mathfrak{B}$ is bounded, all the members of $\mathfrak{B}$ are hermitian in some equivalent norm $\|\cdot \cdot\| \mid$ of $X$ by remarks in $\S 3$ of Lumer [13]. Hence all the members of the closure of the real linear span of $\mathfrak{B}$ are hermitian in $\|\cdot\| \cdot \|$. Now arguing as in the proof of Theorem 3.1 of Berkson [4], it can be shown that each $T$ in $A$ may be written as $T=R+i J$, with $R, J$ in $A$ and hermitian in $\|\cdot\| \cdot \|$. Thus $A$ is a $V^{*}$-algebra in the operator norm $\|\cdot \cdot\|$ induced by the Banach space norm $\|\cdot \mid\|$ of $X$ and hence by Berkson's result (1.2), the theorem follows.

For results on semi-groups of operators one may refer to Hille and Phillips [11].

2. $W^{*}(\|\cdot\|)$-algebras. This section deals with the theory of algebras of operators $W$ on a Banach space $X$ which are generated in the weak operator topology by a $\sigma$-complete Boolean algebra $\mathfrak{B}$ of projections. Such algebras are the natural generalization of abelian von Neumann algebras. The theory of such algebras is developed quite fully in [2], [3] and [9]. It is shown there that by $\overline{\mathfrak{B}}^{s}$;

(i) $W$ is the algebra generated in the uniform operator topology

(ii) $\overline{\mathfrak{B}^{s}}$ is a complete B.A. of projections whose Stone representation space $\mathfrak{m}$ is the maximal ideal space of $W$;

(iii) The Gelfand map $A \rightarrow A($.$) is an isomorphism of W$ onto $C(\mathfrak{m})$ and $W$ and $C(\mathfrak{m})$ are topologically equivalent under this map;

(iv) Every operator $S$ in $W$ is scalar type of class $X^{*} ; S=$ $\int \lambda E(d \lambda)$, whose spectral projections $E(\sigma)$ belong to $\overline{\mathfrak{B}}^{s} \subseteq W$. Further, the space $m$ is extremally disconnected.

Here we make the additional assumption on $W$ that the norm $\|\cdot\|$ on $X$ is such that the operator norm on $W$ is isometric to the supremum norm in $C(\mathfrak{m})$. Such an algebra is called here a $W^{*}(\| \cdot||)$-algebra.

Definition 4. By a $W^{*}(\|\cdot\|)$-algebra $W$ on a Banach space $X$, 
we mean a pair, consisting of a commutative subalgebra $W$ of $\mathfrak{B}(X)$ generated by a $\sigma$-complete B.A. of projections in $X$ in the weak operator topology and some equivalent norm $\|\cdot\|$ on $X$ such that every element $S$ in $W$ has the representation of the form $S=R+i J$ where $R$ and $J$ satisfy the following conditions $(V)$ :
$(V)\left\{\begin{array}{l}(\mathrm{i}) \\ (\mathrm{i})\end{array}\right.$
$R J=J R$ with $R$ and $J$ in $W$;
(ii) $R^{m} J^{n}(m, n=0,1,2 \cdots)$ are hermitian in the norm $\|\cdot\|$.

We make the following observations in regard to a $W^{*}(\| \cdot||)$-algebra.

REMARK 1. A $W^{*}(\|\cdot\|)$-algebra $W$ on a Banach space $X$ is precisely an abelian subalgebra of $\mathfrak{B}(X)$, which is a $V^{*}$-algebra in the operator norm $\|\cdot\|$, induced by the Banach space norm $\|\cdot\|$ on $X$ together with the property that it is generated weakly by a $\sigma$-complete B.A. of projections in $X$.

REMARK 2. A $W^{*}(\|\cdot\|)$-algebra $W$ is a commutative $B^{*}$-algebra in the operator norm $\|\cdot\|$ induced by the Banach space norm $\|\cdot\|$ of $X$ and hence the Gelfand map is an isometric isomorphism of $W$ onto the space $C(\mathfrak{m})$ of complex valued continuous functions, where $\mathfrak{m}$ is the maximal ideal space of $W$.

For, the above remark follows from the fact that a $\sigma$-complete B.A. of projections is bounded and from Theorem (1.3) of $\S 1$.

REMARK 3. The Banach algebra $W$ generated weakly by a $\sigma$-complete B.A. $\mathfrak{B}$ of projections on a Banach space $X$ is a $W^{*}(\|\cdot\|)$-algebra, under a suitable equivalent norm $\|\cdot\|$ on $X$. If $X$ is weakly complete, the hypothesis that $\mathfrak{B}$ is $\sigma$-complete may be replaced by the hypothesis that $\mathfrak{B}$ is bounded.

For, the $\sigma$-completeness of $\mathfrak{B}$ implies that $\overline{\mathfrak{B}}^{s}$ is complete and bounded. Hence the weakly closed algebra generated by $\mathfrak{B}$ coincides with the uniformly closed algebra generated by $\overline{\mathfrak{B}}^{s}$. Now the remark follows by appealing to Theorem (1.3) of $\S 1$.

REMARK 4. An operator $S$ on a Banach space $X$ is scalar type if and only if it belongs to a $W^{*}(\|\cdot\|)$-algebra on $X$.

REMARK 5. If an operator $S$ belongs to a $W^{*}(\|\cdot\|)$-algebra $W$, then $S$ is scalar type and all its spectral projections are in $W$. Further every projection in $W$ is hermitian in $\|\cdot\|$.

Now we shall show that the ${ }^{*}$-operation in a $W^{*}(\|\cdot\|)$-algebra is 
strongly continuous. Though this result is noted in [9] on page 544, the proof that we are giving here is based on the notions of hermiticity and semi-inner-product. In addition, this proof is more direct. To this end, we prove the following lemma.

LEMMA 1. If $E$ is a nonzero projection operator on a Banach space $X$ then there is an equivalent norm $\|\cdot\|$ on $X$ in which $E$ is hermitian and the norm of $E$ computed with respect to $\|\cdot\|$ is unity, i.e., $\|E\|=1$.

Proof. The B.A. $\mathfrak{B}$ of projections, consisting of $0, I, E$ and $I-E^{r}$ is bounded and hence there is an equivalent norm $\|\cdot\|$ on $X$, in which the members of $\mathfrak{B}$ are hermitian. By Theorem 1.3 of $\S 1$, the Banach algeba $\mathscr{E}$ generated by $\mathfrak{B}$ is a $B^{*}$-algebra in the operator norm induced by the Banach space norm $\|\cdot\|$ of $X$. Hence $\|E\|=\sup _{m \in \mathfrak{m}} E(m)=1$ as $E \neq 0$.

THeOREm 1. If $W$ is a $W^{*}(\|\cdot\|)$-algebra on a Banach space $X$, then the involution * defined in $W$ which makes it a $V^{*}$-algebra (see Definition 3 of $\S 1$ ) is continuous in the strong operator topology.

Proof. Since the maximal ideal space $m$ of $W$ is extremally disconnected, every operator $T$ in $W$ admits a spectral representation of the form

$$
T=\int_{\mathfrak{m}} T(m) E(d m)
$$

where $T(m)$ is the Gelfand function associated with $T$ and $E($.$) is a$ strongly countably additive spectral measure, having its range in $W$. Further, for Borel sets $\sigma$ of the maximal ideal space $m$, the projections $E(\sigma)$ are hermitian in $\|\cdot\|$ by Remark 5 and hence $\|E(\sigma)\| \leqq 1$ by Lemma 1.

Let $T_{\alpha}$ in $W$ converge strongly to $T$. Since $W$ is strongly closed, $T$ belongs to $W$. Hence there exist operators $R_{\alpha}, J_{\alpha}, R$ and $J$ in $W$ such that $T=R+i J, T_{\alpha}=R_{\alpha}+i J_{\alpha}$ and $R_{\alpha}, J_{\alpha}$ and $R, J$ satisfy conditions $(V)$ of Definition 4. By following an argument similar to that of Bade (p. 408, [2]), which is available here in view of the spectral representation (1) of any operator $T$ in $W$ and the fact that $\|E(\sigma)\| \leqq 1$ for Borel sets $\sigma$ of the maximal ideal space $\mathfrak{n}$, we can show that $\lim _{\alpha} R_{\alpha}$ and $\lim _{\alpha} J_{\alpha}$ exist in $W$ in the strong operator topology. Let $\lim _{\alpha} R_{\alpha} x=R_{1} x$ and $\lim _{\alpha} J_{\alpha} x=J_{1} x$ for $x$ in $X$.

Now we shall show that $R_{1}=R$ and $J_{1}=J$. If [,] is a semiinner-product on $X$ consistent with the norm $\|\cdot\|$, then, 
for $\|x\|=1$. Since $R_{\alpha}, J_{\alpha}$ are hermitian in $\|\cdot\|,\left[R_{\alpha} x, x\right]$ and $\left[J_{\alpha} x, x\right]$ are real for each $\alpha$ when $\|x\|=1$ and hence $\left[R_{1} x, x\right]$ and $\left[J_{1} x, x\right]$ are real for $\|x\|=1$ by (2). Thus $R_{1}$ and $J_{1}$ are hermitian in $\|\cdot\|$. Similary $R_{1}^{m} J_{1}^{n}(m, n=0,1,2, \cdots)$ are hermitian in $\|\cdot\|$. Hence $R_{1}$ and $J_{1}$ satisfy conditions $(V)$ of Definition 4. Clearly $T x=R_{1} x+i J_{1} x$ for $x \in X$. But $T=R+i J$ by assumption, with $R, J$ satisfying conditions $(V)$. Hence from the uniqueness of the representation of $T$ in $W$, it follows that $R=R_{1}$ and $J=J_{1}$.

Now $\lim T_{\alpha}^{*} x=\lim \left(R_{\alpha}-i J_{\alpha}\right) x=\left(R_{1}-i J_{1}\right) x=(R-i J x)=T^{*} x$ for $x$ in $X$. This establishes the strong continuity of the involution *

In the rest of this section we generalize the notion of positivity of operators on a Hilbert space to operators on a Banach space. We recall that in a Hilbert space $H$, an operator $T$ is called positive if $(T x, x) \geqq 0$ for $x$ in $H$, where (, ) is the inner-product of $H$. Also it is known there that $T$ is positive if and only if $\sigma(T)$ is nonnegative.

Definition 5. An operator $T$ on a Banach space $X$ is called positive in the equivalent norm $\|\cdot\|$ on $X$ (which we denote by $T \geqq 0$ in $\|\cdot\|)$ if $[T x, x] \geqq 0$ for $x$ in $X$, with $\|x\|=1$, where [,] is a semiinner-product consistent with the norm $\|\cdot\|$ on $X$; i.e., if the numerical range $W(T)$ with respect to the semi-inner-product [,] is nonnegative.

The above definition calls for several comments. Since there may be an infinite number of semi-inner-products consistent with a given norm, the definition looks ambiguous at first sight. But the ambiguity disappears in the light of Theorem 14 of Lumer [12], according to which the numerical range has the same convex hull relative to any two semi-inner-products inducing the same norm. It may be noted that this definition also coincides with the classical one in a Hilbert space.

Lemma 2. If $E$ is a projection operator on a Banach space $X$ and is hermitian in the equivalent norm $\|\cdot\|$ on $X$ then $E$ is positive in $\|\cdot\|$.

Proof. The cases in which $E=0$ or $E=I$ are trivial. Hence suppose $E \neq 0, I$. Then by Lemma $1\|E\|=1$ and $\|I-E\|=1$, since $E$ and $I-E$ are hermitian in $\|\cdot\|$. Now for $x$ in $X$ with $\|x\|=1$ and a semi-inner- product [, ] consistent with the norm $\|\cdot\|$, we have 


$$
\begin{aligned}
{[E x, x] } & =[\{I-(I-E)\} x, x] \\
& =1-[(I-E) x, x] \\
& \geqq 0
\end{aligned}
$$

as $[(I-E) x, x]$ is real and $|[(I-E) x, x]| \leqq\|I-E\|=1$. Hence the lemma.

The above lemma on projections enables us to link the positivity of an operator in a $W^{*}(\|\cdot\|)$-algebra, with the nonnegativeness of its spectrum.

THEOREM 2. If $T$ is an operator belonging to a $W^{*}(\|\cdot\|)$-algebra $W$ then the following are equivalent.

(i) $\sigma(T)$ is nonnegative.

(ii) The Gelfand function $T(m)$ in $C(\mathrm{nt})$ is nonnegative where $\mathrm{nt}$ is the maximal ideal space of $W$.

(iii) $T$ is positive in $\|\cdot\|$.

Proof. The equivalence of (i) and (ii) is clear from the results that the spectrum of $T$ in $W$, viz. $\sigma_{W}(T)$, is the range of $T(m)$ and that $\sigma_{W}(T)=\sigma(T)$ (see Corollary 3.7.6 of Rickart [16]).

To prove the theorem, therefore it suffices to show that (i) and (iii) are equivalent. Let (i) hold. Then, as $T$ belongs to the $W^{*}(\|\cdot\|)$ algebra $W$ it is scalar type and its spectral projections are in $W$. Further they are hermitian in $\|\cdot\|$ by Remark 5 . Hence, if $E($.$) is$ the resolution of the identity of $T$, then $E(\sigma)$ are hermitian in $\|\cdot\|$ for Borel sets $\sigma$ of the complex plane, so that $E(\sigma)$ are positive in $\|\cdot\|$ by Lemma 2. Now let [, ] be a semi-inner-product on $X$ consistent with the norm $\|\cdot\|$. Then for $x$ in $X$, with $\|x\|=1,[E() x, x$.$] is a$ positive measure and hence

$$
\begin{aligned}
{[T x, x] } & =\left[\int_{\sigma(T)} \lambda E(d \lambda) x, x\right] \\
& =\int_{\sigma(T)} \lambda[E(d \lambda) x, x] \\
& \geqq 0
\end{aligned}
$$

as $\sigma(T)$ is nonnegative. Hence $T$ is positive in $\|\cdot\|$; i.e., (iii) holds.

Conversely, let (iii) hold. Then as $T$ is in $W, T$ is scalar type. Hence by Theorem 5, $§ 4$, of Foguel [10], $\sigma(T)=\pi(T)$ where $\pi(T)$ is the approximate point spectrum of $T$. Since $T$ is bounded, by Theorem 4 of Lumer [12], we have $\pi(T) \subseteq \overline{W(T)}$ where $W(T)$ is the numerical range of $T$ with respect to some semi-inner-product consistent with the norm $\|\cdot\|$. But by hypothesis $W(T)$ is nonnegative and hence $\overline{W(T)}$ is nonnegative. Hence $\sigma(T)=\pi(T)$ is nonnegative. Therefore (i) holds.

This completes the proof of the theorem. 
Definition 6. For two operators $T, T^{\prime}$ on a Banach space $X$ we say $T$ is greater than $T^{\prime}$ in the equivalent norm $\|\cdot\|$ on $X$ (briefly $T \geqq T^{\prime}$ in $\|\cdot\|$ ) if (i) $T, T^{\prime}$ are hermitian in $\|\cdot\|$ and (ii) $T-T^{\prime}$ is positive in $\|\cdot\|$.

Definition 7. A net $\left\{T_{\alpha}\right\}$ is said to be monotonic increasing (decreasing) in the equivalent norm $\|\cdot\|$ on $X$ if $T_{\alpha} \geqq T_{\beta}\left(T_{\beta} \geqq T_{\alpha}\right)$ in $\|\cdot\|$ whenever $\alpha \geqq \beta$. In symbols we write this as $\left\{T_{\alpha}\right\}$ m.i. (m.d.) in $\|\cdot\|$.

We recall that in a Hilbert space, if $\left\{T_{\alpha}\right\}$ is a bounded monotonic net of commuting hermitian operators, then $\left\{T_{\alpha}\right\}$ converges strongly to a Hermitian operator. We generalize this result to Banach spaces below.

Lemma 3. Let $T_{\alpha} \downarrow 0$ be a monotonic decreasing net in a $W^{*}(\|\cdot\|)$ algebra $W$. Then $T_{\alpha} \rightarrow 0$ in the strong operator topology.

Proof. ${ }^{1} \quad$ By Theorem 2, for elements $S_{1}$ and $S_{2}$ in $W, S_{1} \geqq S_{2}$ in $\|\cdot\|$ if and only if $S_{1}(m) \geqq S_{2}(m)$ in $C(m)$. Since $m$ is stonean, the hermitian elements of $C(\mathfrak{m})$ and hence of $W$ form a conditionally complete lattice under this partial ordering.

For $\alpha \geqq \alpha_{0}, T_{\alpha} \leqq T_{\alpha_{0}}$ in $\|\cdot\|$. Hence $T_{\alpha}(m) \leqq T_{\alpha_{0}}(m)$ so that $\left\|T_{\alpha}\right\|=$ $\sup \left|T_{\alpha}(m)\right|=\sup T_{\alpha}(m) \leqq \sup T_{\alpha_{0}}(m)=\left\|T_{\alpha_{0}}\right\|$. Hence

$$
\left\|T_{\alpha}\right\| \leqq\left\|T_{\alpha_{0}}\right\|
$$

for $\alpha \geqq \alpha_{0}$.

Let $x \in X$ and $\varepsilon>0$. If $e_{\alpha}=\left\{m: T_{\alpha}(m)<\varepsilon\right\}$ then $\bigvee_{\alpha} E\left(e_{\alpha}\right)=I$ so that $E\left(e_{\alpha}\right) \rightarrow I$ strongly where $E($.$) is a countably additive spectral$ measure on the Borel sets of $m$ with respect to which $T_{\alpha}$ have the spectral representation

$$
T_{\alpha}=\int_{\mathfrak{m}} T_{\alpha}(m) E(d m)
$$

Thus for $x \in X$,

$$
\left\|T_{\alpha} x\right\| \leqq\left\|T_{\alpha} E\left(e_{\alpha}\right) x\right\|+\left\|T_{\alpha}\right\|\left\|\left(I-E\left(e_{\alpha}\right)\right) x\right\|<\varepsilon\|x\|+\varepsilon
$$

for $\alpha \geqq \alpha_{0}$ in view of (3) and Lemma 1 . Thus $T_{\alpha} \rightarrow 0$ strongly.

THEOREM 3. Let $\left\{T_{\alpha}\right\}$ be a net in a $W^{*}(\|\cdot\|)$-algebra $W$ of operators on a Banach space $X$ such that

(i) $\left\{T_{\alpha}\right\}$ is monotonic in $\|\cdot\|$; and

(ii) for some $R$ in $W, T_{\alpha} \leqq R$ in $\|\cdot\|$ if $\left\{T_{\alpha}\right\}$ is m.i. in $\|\cdot\|$ and

1 The present short proof is due to the referee to whom the author is thankful. 
$T_{\alpha} \geqq R$ in $\|\cdot\|$ if $\left\{T_{\alpha}\right\}$ is m.d. in $\|\cdot\|$; i.e., the net $\left\{T_{\alpha}\right\}$ is bounded. Then $\lim _{\alpha} T_{\alpha} x$ exists for each $x$ in $X$. Further $\lim _{\alpha} T_{\alpha} x=\bigvee_{\alpha} T_{\alpha} x$ $\left(\bigwedge_{\alpha} T_{\alpha} x\right)$ if $\left\{T_{\alpha}\right\}$ is m.i. (m.d.) in $\|\cdot\|$.

Proof. Without loss of generality we may assume that $\left\{T_{\alpha}\right\}$ is m.i. in $\|\cdot\|$. Since $T_{\alpha} \leqq R$ in $\|\cdot\|$ and since the hermitian elements in $W$ form a conditionally complete lattice, it follows that $T_{\alpha} \uparrow V_{\alpha} T_{\alpha}$ in $W$. Hence $\left(\mathrm{V}_{\alpha} T_{\alpha}-T_{\alpha}\right) \downarrow 0$. Then it follows from Lemma 3 that $\lim _{\alpha} T_{\alpha} x=\mathrm{V}_{\alpha} T_{\alpha} x$ for $x$ in $X$. Hence the theorem.

3. Unbounded spectral operators of scalar type. In this section we obtain some interesting results on unbounded spectral operators of scalar type, which will be needed in the sequel. For definitions and results on such operators which are used here, the reader may refer to Bade [1].

Let $\mathfrak{m}$ be a set and $\Sigma$ be a $\sigma$-algebra of subsets of $\mathfrak{m}$. Let $E($. be an $X^{*}$-countably additive spectral measure on $\Sigma$. Suppose $f$ is a complex valued $E($.)-essentially unbounded $\Sigma$-measurable function on $\mathfrak{m}$. Then we define a linear transformation $f(E)$ as below.

Definition 8. Let $e_{n}=\{m: m \in \mathfrak{m},|f(m)| \leqq n\}$. Then define $f_{n}$ as follows.

$$
\begin{aligned}
f_{n}(m) & =f(m), m \in e_{n} \\
& =0, m \notin \boldsymbol{e}_{n} .
\end{aligned}
$$

We define

$$
D(f)=\left\{x: x \in X \text { and } \lim _{n \rightarrow \infty} \int_{e_{n}} f(m) E(d m) x \text { exists }\right\}
$$

and

$$
f(E) x=\lim _{n \rightarrow \infty} \int_{e_{n}} f(m) E(d m) x, x \in D(f) .
$$

It is easy to check that $D(f)$ is a dense linear manifold in $X$ and $f(E)$ is a linear transformation over $D(f)$ with its range in $X$.

Lemma 4. Let $A(f)$ be the set of members in $\Sigma$ on which $f$ is bounded. Then we have:

(i) $A(f)$ is closed under finite unions and contains any subset of its members, if the subset belongs to $\Sigma$;

(ii) If $e \in A(f)$, then $E(e) X \subseteq D(f)$ and $f(E)$ is bounded in $E(e) X$, where $D(f)$ and $f(E)$ are as in Definition 8;

(iii) $E(e) f(E) E(e)=f(E) E(e), e \in A(f)$;

(iv) $A(f)$ contains an increasing sequence $\left\{\delta_{n}\right\}$ such that

$$
E\left(\bigcup_{n=1}^{\infty} \delta_{n}\right)=I \text {. }
$$


Proof. The statement (i) is obvious. The statement (iv) is clear, if we take for $\left\{\delta_{n}\right\}$ the sequence $\left\{e_{n}\right\}$ in Definition 8 .

Since $f$ is bounded on $e \in A(f)$ and since $\lim _{n \rightarrow \infty} E\left(e_{n}\right) x=x$ for $x \in X$, we have

$$
\begin{aligned}
f(E) E(e) x & =\lim _{n \rightarrow \infty} \int_{e_{n}} f(m) E(d m) E(e) x \\
& =\lim _{n \rightarrow \infty} \int_{e} f(m) E(d m) E\left(e_{n}\right) x \\
& =\int_{e} f(m) E(d m) x .
\end{aligned}
$$

Now from (4) the assertions (ii) and (iii) follow.

Lemma 5. Let $\left\{\delta_{n}\right\}$ be any other increasing sequence from $A(f)$ for which $E\left(\cup_{n=1}^{\infty} \delta_{n}\right)=I$. Then:

(i) $\lim _{n \rightarrow \infty} f(E) E\left(e_{n}\right) x$ exists if and only if $x$ is in $D(f)$;

(ii) If $\lim _{n \rightarrow \infty} f(E) E\left(e_{n}\right) x$ exists, then

$$
\lim _{n \rightarrow \infty} f(E) E\left(e_{n}\right) x=\lim _{n \rightarrow \infty} f(E) E\left(\delta_{n}\right) x
$$

and conversely;

(iii) If in the definition of $f(E)$ the sequence $\left\{e_{n}\right\}$ is replaced by any other sequence $\left\{\delta_{n}\right\}$ in $A(f)$ such that $\delta_{n} \supseteqq \delta_{n-1}$ and $E\left(\bigcup_{n=1}^{\infty} \delta_{n}\right)=I$, we obtain the same linear transformation $f(E)$.

Proof. Let $x$ be in $X$. Then by relation (4) in the above, we have

$$
f(E) E\left(e_{n}\right) x=\int_{e_{n}} f(m) E(d m) x
$$

from which the assertion (i) of the lemma follows.

The statement (ii) of the lemma can be proved by following an argument similar to that of Lemma 2.1 of Bade [1].

Finally, to prove (iii) let $x$ be in $D(f)$. Then by relation (4) and statement (ii) of the lemma we have

$$
\begin{aligned}
f(E) x & =\lim _{n \rightarrow \infty} \int_{e_{n}} f(m) E(d m) x \\
& =\lim _{n \rightarrow \infty} f(E) E\left(e_{n}\right) x \\
& =\lim _{n \rightarrow \infty} f(E) E\left(\delta_{n}\right) x \\
& =\lim _{n \rightarrow \infty}\left(\lim _{k \rightarrow \infty} \int_{e_{k}} f(m) E(d m)\right) E\left(\delta_{n}\right) x \\
& =\lim _{n \rightarrow \infty} \lim _{k \rightarrow \infty} \int_{e_{k} \cap \delta_{n}} f(m) E(d m) x \\
& =\lim _{n \rightarrow \infty} \lim _{k \rightarrow \infty} E\left(e_{k}\right) \int_{\delta_{n}} f(m) E(d m) x
\end{aligned}
$$


so that

$$
f(E) x=\lim _{n \rightarrow \infty} \int_{\delta_{n}} f(m) E(d m) x .
$$

Now the assertion (iii) is clearly a consequence of equation (5) and statements (i) and (ii) of the lemma. Hence the lemma.

As a consequence of the above lemmas, we prove the following main theorem of this section. We also remark that this theorem is a generalization of Lemma 6 of Dunford [8], to the unbounded case.

THEOREM 4. Let $f$ be a complex valued $E(\cdot)$-essentially unbounded $\Sigma$-measurable function on the set $\mathrm{m}$, where $E(\cdot)$ is an $X^{*}$-countably additive spectral measure on $\Sigma$, a $\sigma$-algebra of subsets of $\mathrm{m}$. Then:

( i ) The set $D(f)=\left\{x: \lim _{n \rightarrow \infty} \int_{e_{n}} f(m) E(d m) x\right.$ exists $\}$ is a dense linear manifold of $X$ where

$$
e_{n}=\{m: m \in \mathfrak{M},|f(m)| \leqq n\} ;
$$

(ii) The operator $f(E)$ defined by

$$
f(E) x=\lim _{n \rightarrow \infty} \int_{e_{n}} f(m) E(d m) x
$$

is an unbounded spectral operator of scalar type with domain $D(f)$ whose resolution of the identity is given by $E_{f}(\cdot)$ where

$$
E_{f}(\sigma)=E\left(f^{-1}(\sigma)\right)
$$

for Borel sets $\sigma$ of the complex plane;

(iii) Any other increasing sequence $\left\{\delta_{n}\right\}$ in $A(f)$ (see Lemma 4 for definition of $A(f)$ ) such that $E\left(\mathbf{U}_{n=1}^{\infty} \delta_{n}\right)=I$ would also define the same linear transformation $f(E)$.

Proof. The proof of (i) is trivial. The assertion (iii) follows from Lemma 5.

To prove (ii), let $E_{f}(\cdot)=E\left(f^{-1}(\cdot)\right)$ on the family $\mathfrak{B}$ of Borel sets of the complex plane. $E_{f}(\cdot)$ is also an $X^{*}$-countably additive spectral measure. Defining $f_{n}$ as in Definition 8 , we have

$$
\int_{e_{n}} f(m) E(d m)=\int_{\mathfrak{m}} f_{n}(m) E(d m)=\int_{\frac{f_{n}(\mathfrak{m})}{}} \lambda E_{f_{n}}(d \lambda)
$$

by Lemma 6 of Dunford [8], where $E_{f_{n}}(\cdot)=E\left(f_{n}^{-1}(\cdot)\right)$.

Now by Lemma $1, \S 3$, of Foguel [10], the operator $\int_{\overline{f_{n}(\mathfrak{m})}} \lambda E_{f_{n}}(d \lambda)$ belongs to the uniformly closed algebra generated by $E_{f_{n}}(\alpha)$ for Borel 
sets $\alpha$ of the complex plane such that $0 \notin \bar{\alpha}$. Let $\alpha$ be such a Borel set of $\overline{f_{n}(\mathfrak{m})}$. Since $\alpha \nexists 0$

$$
f_{n}^{-1}(\alpha)=\left\{m ; f(m) \in \alpha \text { and } m \in e_{n}\right\}
$$

as $f_{n}=f$ on $e_{n}$. But, as $\overline{f_{n}(\mathfrak{m})} \leqq\{\lambda:|\lambda| \leqq n\}, \alpha \subseteq \overline{f_{n}(\mathfrak{m})} \leqq\{\lambda:|\lambda| \leqq n\}$. Hence $f^{-1}(\alpha) \subseteq e_{n}$. Thus

$$
f^{-1}(\alpha)=\left\{m: f(m) \in \alpha \text { and } m \in e_{n}\right\} .
$$

Hence from (7) and (8) it follows that $f_{n}^{-1}(\alpha)=f^{-1}(\alpha)$ so that $E_{f_{n}}(\alpha)=$ $E_{f}(\alpha)$. Hence by the above lemma of Foguel [10] we have

$$
\begin{aligned}
\int_{e_{n}} f(m) E(d m) & =\int_{\overline{f_{n}(m)}} \lambda E_{f_{n}}(d \lambda) \\
& =\int_{\overline{f\left(e_{n}\right)}} \lambda E_{f_{n}}(d \lambda) \\
& =\int_{\overline{f\left(e_{n}\right)}} \lambda E_{f}(d \lambda)
\end{aligned}
$$

as $f=f_{n}$ on $e_{n}$ and $f_{n}(m)=0$ for $m \notin e_{n}$. Thus,

$$
\begin{aligned}
f(E) x & =\lim _{n \rightarrow \infty} \int_{e_{n}} f(m) E(d m) x \\
& =\lim _{n \rightarrow \infty} \int_{\frac{f\left(e_{n}\right)}{}} \lambda E_{f}(d \lambda) x .
\end{aligned}
$$

Clearly the left hand side and the right hand side of (9) exist if and only if $x$ is in $D(f)$. Hence the right hand side of (9) defines the same operator $f(E)$.

Now as $\overline{f\left(e_{n}\right)} \leqq\{\lambda:|\lambda| \leqq n\}$, the sequence $\overline{\left\{f\left(e_{n}\right)\right\}}$ is a sequence of bounded closed sets in the complex plane. Clearly it is an increasing sequence. Also it is easy to check that $E_{f}\left(\bigcup_{n=1}^{\infty} \overline{f\left(e_{n}\right)}\right)=I$. Hence by Theorem 3.3. of Bade [1] and by the definition of scalar type operators (see p. 379, Bade [1]) it follows that the operator $f(E)$ defined by

$$
f(E) x=\lim _{n \rightarrow \infty} \int_{\overline{f\left(e_{n}\right)}} \lambda E_{f}(d \lambda) x, x \in D(f)
$$

is an unbounded spectral operator of scalar type with the resolution of the identity $E_{f}(\cdot)$.

This completes the proof of the theorem.

In Theorem 3.3 of Bade [1] the operator $f(S)$ (see p. 379 of Bade [1] for definition) is proved to be spectral and nothing has been said whether $f(S)$ is scalar type. But the above theorem asserts that $f(S)$ is scalar type and hence we state this result separately below.

Corollary. If $f$ is an E(-)-essentially unbounded Borel measu- 
rable function over the complex plane where $E(\cdot)$ is an $X^{*}$-countably additive spectral measure over the Borel sets of the complex plane, then the operator $f(S)$ is an unbounded spectral operator of scalar type.

In [10] Foguel has proved that the residual spectrum of a (bounded) spectral operator of finite type is empty. In the following theorem we generalize this result to unbounded spectral operators of scalar type.

THEOREM 5. Let $S$ be an unbounded spectral operator of scalar type on a Banach space $X$, with its resolution of the identity $E(\cdot)$. Then a point $l$ in the complex plane belongs to (i) the point spectrum $\sigma_{P}(S)$ if and only if $E(l) \neq 0$ and (ii) the continuous spectrum $\sigma_{c}(S)$ if and only if $l \in \sigma(S)$ and $E(l)=0$. Consequently, the residual spectrum of $S$ is empty.

Proof. ${ }^{2}$ Suppose $E(l)=0$. Then the function $f(\lambda)=(\lambda-l)^{-1}$ is analytic and single valued in the complement of the single point closed set $l$ for which $E(l)=0$. Hence $f(\lambda) \in \Re$ (see p. 387 of Bade [1] for definition of $\Re$ ). Now taking

$$
e_{n}=\left\{\lambda:|\lambda| \leqq n, \text { dist. }(\lambda, l) \geqq \frac{1}{n}\right\}
$$

$\left\{e_{n}\right\}$ is an increasing sequence of bounded closed sets for which

$$
E\left(\bigcup_{n=1}^{\infty} e_{n}\right)=I \text {. }
$$

Therefore defining

$$
f(S) x=\lim _{n \rightarrow \infty} \int_{e_{n}} f(\lambda) E(d \lambda) x
$$

on the set $D(f(S))$ of $x$ for which the limit in (10) exists, we see that $f(S)$ is a closed operator with its domain $D(f(S))$ dense in $X$.

Now for $x$ in $D(f(S))$ we have

$$
\begin{aligned}
& \int_{e_{n}}(\lambda-l) E(d \lambda) f(S) x \\
= & \int_{e_{n}}(\lambda-l) E(d \lambda) \lim _{k \rightarrow \infty} \int_{e_{k}} f(\lambda) E(d \lambda) x \\
= & \lim _{k \rightarrow \infty} \int_{e_{n}}(\lambda-l) E(d \lambda)\left[\int_{e_{n}} f(\lambda) E(d \lambda) x+\int_{e_{k}-e_{n}} f(\lambda) E(d \lambda) x\right] \\
= & \int_{e_{n}}(\lambda-l) f(\lambda) E(d \lambda) x=E\left(e_{n}\right) x .
\end{aligned}
$$

2 The method we adopt here is an extension of that on p. 325 of Stone [17] for maximal normal operators on Hilbert spaces. 
Hence for $x$ in $D(f(S))$ we have

$$
(S-l I) f(S) x=\lim _{n} E\left(e_{n}\right) x=x .
$$

Similarly we have for $x$ in $D(S)=D(S-l I), f(S)(S-l I) x=x$. Thus $f(S)$ is the inverse $(S-l I)^{-1}$. From (11) and that $D(f(S))$ is dense in $X$ it follows that the range of $S-l I$ is dense in $X$. Hence $l$ is not in the residual spectrum of $S$. Also as $(S-l I)^{-1}$ exists, $l$ is not in the point spectrum $\sigma_{P}(S)$. Thus if $l$ belongs to $\sigma(S)$ and $E(l)=0$ then $l \in \sigma_{c}(S)$. This proves the direct part of assertion (ii). The converse part of (ii) clearly follows from the result (i), which we shall now prove.

Suppose $l \in \sigma_{P}(S)$. If $E(l)=0$ then from the above, we must have $l$ in the continuous spectrum $\sigma_{c}(S)$ which is a contradiction as

$$
\sigma_{P}(S) \cap \sigma_{c}(S)=\varphi \text {. }
$$

Hence $E(l) \neq 0$.

Conversely, suppose $E(l) \neq 0$. Clearly $l \in \sigma(S)$. Since $E(l) \neq 0$ there is a vector $x_{0} \in X$ such that $E(l) x_{0} \neq 0$. Then

$$
S E(l) x_{0}=\lim _{n \rightarrow \infty} \int_{\sigma_{n}} \lambda E(d \lambda) E(l) x_{0}
$$

where $\sigma_{n}=\{\lambda:|\lambda| \leqq n\}$. Therefore

$$
\begin{aligned}
S E(l) x_{0} & =\lim _{n \rightarrow \infty} \int_{\sigma_{n} \cap\{l\}} \lambda E(d \lambda) x_{0} \\
& =\int_{\{l\}} \lambda E(d \lambda) x_{0}=l E(l) x_{0}
\end{aligned}
$$

and since $E(l) x_{0} \neq 0, l$ is in $\sigma_{P}(S)$.

This completes the proof of the theorem.

The following theorem on $W^{*}(\|\cdot\|)$-algebras which plays a key role in the spectral representation of semi-groups of scalar type operators, is a consequence of the preceding results.

Theorem 6. Let $W$ be a $W^{*}(\|\cdot\|)$-algebra. If $\mathrm{m}$ is the maximal ideal space of $W$, then there exists an $X^{*}$-countably additive spectral measure $P(\cdot)$ on the Borel sets $\Sigma$ of $m$ such that each bounded Borel measurable function $f$ on $m$ corresponds to a unique operator $F$ in $W$, the correspondence being given by

$$
F=\int_{\mathfrak{m}} f(m) d_{m} P(E)^{3}
$$

${ }^{3} \int_{\mathfrak{n}} f(m) P(d m)$ is denoted by $\int_{\mathfrak{m}} f(m) d_{m} P(E)$ hereafter following the notation of Hille and Phillips [11]. 
the above integral existing in the uniform operator topology.

Also if $f$ is a $P(\cdot)$-essentially unbounded Borel measurable function on $\mathrm{m}$ and if $\left\{e_{n}\right\}$ is an increasing sequence of Borel sets of $m$ on which $f$ is bounded and if $P\left(\bigcup_{n=1}^{\infty} e_{n}\right)=I$ then the set $D(f)$ of all $x$ of $X$ for which

$$
f(P) x=\lim _{n \rightarrow \infty} \int_{e_{n}} f(m) d_{m} P(E) x
$$

exists, is dense in $X$ and the transformation $f(P)$ defined by (12) is an unbounded spectral operator of scalar type with domain $D(f)$. Consequently, the residual spectrum of $f(P)$ is empty.

REMARK 6. If the Borel measurable function $f$ in the above theorem is real valued, an equivalent representation for $f(P)$ in (12) is obtained as follows.

If $E_{\lambda}=\{m: f(m) \leqq \lambda\}$, then denote the projection $P\left(E_{\lambda}\right)$ by $P(\lambda)$. The family of projections $[P(\lambda):-\infty<\lambda<\infty]$ which generates the resolution of the identity $P_{f}(\cdot)$ of $f(P)$ (see Theorem 4) has the following properties $(\psi)$.

$$
(\psi) \begin{cases}\text { ( i ) } & \wedge P(\lambda)=0, \vee P(\lambda)=I ; \\ \text { (ii) } & P(\lambda) P(\mu)=P(\lambda) \text { for } \lambda \leqq \mu ; \\ \text { (iii) } & P(\lambda)=\bigwedge_{\mu>\lambda} P(\mu) .\end{cases}
$$

Also the property (iii) is equivalent to

(iii) $\quad P(\lambda) x=\lim _{t^{\prime \rightarrow \lambda}+\lambda^{+}} P(\mu) x, x \in X$ in view of Lemma 2.3 of Bade [3].

Further, by Theorem 4 the equation (12) can be written as

$$
f(P) x=\lim _{n \rightarrow \infty} \int_{\sigma_{n}} \lambda d P_{f}(\lambda) x, x \in D(f)
$$

where $P_{f}(\cdot)=P\left(f^{-1}(\cdot)\right)$ and $\left\{\sigma_{n}\right)$ is any increasing sequence of bounded Borel sets of the real line such that $P_{f}\left(\bigcup_{n=1}^{\infty} \sigma_{n}\right)=I$.

Since the resolution of the identity $P_{f}(\cdot)$ of $f(P)$ is generated by the family $[P(\lambda):-\infty<\lambda<\infty]$, (12) is written as

$$
f(P) x=\int_{-\infty}^{\infty} \lambda d P(\lambda) x, x \in D(f) \text {. }
$$

4. Semi-groups of real scalar type operators. In this section we obtain the spectral representation of a strongly measurable (and hence strongly continuous) semi-groups of real scalar type operators. We also obtain an ergodic theorem for such semi-groups of operators. Henceforth we closely follow the notations of Hille and Phillips [11] 
and often omit details of analogous proofs.

DeFinition 9. Let $\subseteq \equiv[T(\xi): \xi>0]$ be a semi-group of operators on $X$. Then by $X_{0}$ we denote the set $\bigcup_{\xi>0} T(\xi) X$. (see p. 307 of [11]).

THEOREM 7. Let $\mathfrak{S} \equiv[T(\xi): \xi>0]$ be a strongly measurable semigroup of real scalar type operators of class $X^{*}$ on $X$ and let the members of $\subseteq$ belong to a $W^{*}(\|\cdot\|)$-algebra $W$. Then $\subseteq$ is of finite type $\omega_{0}$ (say) and $\|T(\xi)\|=\exp \left(\omega_{0} \xi\right)$. Also,

$$
\lim _{\xi \rightarrow 0^{+}} T(\xi) x=J x, x \in X
$$

where $J$ is a projection with its range $\Re(J)=\bar{X}_{0}$ and

$$
T(\xi) J=J T(\xi)=T(\xi)
$$

for all $\xi>0$.

Finally, $T(\xi)$ has a holomorphic extension $T(\tau)$ having either the whole plane or the right half-plane as its maximal domain of analytic existence and there exists a unique representation of $T(\tau)$ of the form

$$
T(\tau) x=\int_{-\infty}^{\omega_{0}} e^{\lambda \tau} d P(\lambda) x, x \in X .
$$

Here $[P(\lambda)]$ generates the resolution of the identity relative to $\bar{X}_{0}$ for the infinitesimal operator $A_{0}$ of $\subseteq$, viz.,

$$
A_{0} x=\int_{-\infty}^{\omega_{0}} \lambda d P(\lambda) x
$$

where $D\left(A_{0}\right)$, the domain of $A_{0}$, coincides with the set of all $x \in \bar{X}_{0}$ for which the integral in (14) exists. Also $D\left(A_{0}\right)$ is dense in $\bar{X}_{0}$ and $A_{0}$ is an unbounded spectral operator of real scalar type on $\bar{X}_{0}$.

Proof. That the semi-group $\subseteq$ is of finite type $\omega_{0}$ follows from the facts that (i) $W$ is an abelian $B^{*}$-algebra in the norm $\|\cdot\|$ (ii) $\|T(\xi)\|$ is lower-semi-continuous and (iii) $\subseteq$ is a nontrivial semi-group. Also it follows that $\|T(\xi)\|=e^{\omega_{0} \xi}$.

Since the maximal ideal space $\mathfrak{M}$ of $W$ is extremally disconnected and $\|T(\xi)\|=e^{\omega_{0} \xi}$ the argument on p. 589 of Hille and Phillips [11] can be applied verbatim, taking $W$ for $\mathfrak{B}$ there. Defining $\mathfrak{W}, \mathfrak{U}$ and $\alpha(m)$ in the same way as in [11] and putting $P(\lambda)=P\left(E_{\lambda}\right)=P\{m: \alpha(m) \leqq \lambda\}$ it is easy to see that $[P(\lambda)]$ generates a resolution of the identity relative to the subspace $\Re[P(\mathfrak{W})]$; i.e., $P(\lambda)$ satisfies conditions $(\psi)$ in $\S 3$ except that $\bigvee P(\lambda)=P(\mathfrak{W})=J . \quad J$ may differ from $I$. Then arguing as in [11] we have 


$$
T(\xi) x=\int_{\mathfrak{M}} T(\xi)(m) d_{m} P(E) x=\int_{-\infty}^{\omega_{0}} e^{\lambda \xi} d P(\lambda) x .
$$

Consequently,

$$
x^{*} e^{-\omega_{0} \xi} T(\xi) x=\int_{-\infty}^{0} e^{\lambda \xi} x^{*} d P\left(\lambda+\omega_{0} \xi\right) x
$$

for each $x \in X$ and $x^{*} \in X^{*}$. Since $x^{*} P(\lambda) x$ is continuous in the right for fixed $x^{*}$ and $x$, by arguing in the same way as on p. 590 of [11] it can be shown that $[P(\lambda)]$ is uniquely determined by $\mathfrak{S}$. Now

$$
\begin{aligned}
\left\|\int_{-\infty}^{\beta} e^{\lambda \xi} d P(\lambda) x\right\| & =\left\|\int_{\mathfrak{W}_{\beta}} e^{\xi \alpha(m)} d_{m} P(E) x\right\| \\
& =\left\|\int_{\mathfrak{W}_{\mathfrak{B}}} e^{\xi \alpha(m)} d_{m} P(E) P\left(\mathfrak{W}_{\beta}\right) x\right\| \\
& =\left\|\int_{\mathfrak{M}} T(\xi)(m) d_{m} P(E) P\left(\mathfrak{W}_{\beta}\right) x\right\|
\end{aligned}
$$

where $\mathfrak{W}_{\beta}=\{m: m \in \mathfrak{M}, \alpha(m) \leqq \beta\}$.

Hence by Lemma 6 of Dunford [8] and Lemma 1 of $\S 2$,

$$
\begin{aligned}
\left\|\int_{-\infty}^{\beta} e^{\lambda \xi} d P(\lambda) x\right\| \leqq & 4 \sup _{m \in \mathfrak{m}} \mid T(\xi)(m)\|\| P\left(\mathfrak{W}_{\beta}\right) x \| \\
= & 4 e^{\omega_{0} \xi}\left\|P\left(\mathfrak{W}_{\beta}\right) x\right\| \\
& <4 \max \left\{1, e^{\omega_{0} t}\right\}\|P(\beta) x\| \\
& <\varepsilon
\end{aligned}
$$

for $\xi$ in $[0, t]$ if $\beta<-N(\varepsilon)$, as $\|P(\beta) x\| \rightarrow 0$ as $\beta \rightarrow-\infty$. Hence $\lim _{\beta \rightarrow-\infty} \int_{-\infty}^{\beta} e^{\lambda \xi} d P(\lambda) x=0$ uniformly for $\xi$ in $[0, t]$ so that the double limit

$$
\lim _{\substack{\xi \rightarrow 0^{+} \\ \beta \rightarrow-\infty}} \int_{\beta}^{\omega_{0}} e^{\lambda \xi} d P(\lambda) x
$$

exists and hence the two iterated limits exist and are equal. Thus

$$
\begin{aligned}
\lim _{\xi \rightarrow 0^{+}} T(\xi) x & =\lim _{\xi \rightarrow 0^{+}} \lim _{\beta \rightarrow-\infty} \int_{\beta}^{\omega_{0}} e^{\lambda \xi} d P(\lambda) x \\
& =\lim _{\beta \rightarrow-\infty} \lim _{\xi \rightarrow 0^{+}} \int_{\beta}^{\omega_{0}} e^{\lambda \hat{\xi}} d P(\lambda) x=J x
\end{aligned}
$$

for $x \in X$. Hence the equation (13).

The argument on p. 590 in [11] holds here to show that $\Re(J)=\bar{X}_{0}$ and $J T(\xi)=T(\xi) J=T(\xi)$. Further, the part concerning the holomorphic extension can be proved arguing in the same way as in [11], replacing $(P(\lambda) x, y)$ there by $x^{*} P(\lambda) x, x \in X$ and $x^{*} \in X^{*}$.

Define the operator $A$ as follows. 


$$
A x=\lim _{n \rightarrow \infty} \int_{e_{n}} \alpha(m) d_{m} P(E) J x
$$

where $e_{n}=\{m: m \in \mathfrak{W},|\alpha(m)| \leqq n\}$, for all $x$ in $\bar{X}_{0}$ for which the limit in (16) exists. Since $P\left(\bigcup_{n=1}^{\infty} e_{n}\right) J=P(\mathfrak{W}) J=J, A$ is an unbounded spectral operator of real scalar type on $\bar{X}_{0}$ and

$$
A x=\int_{-\infty}^{w_{0}} \lambda d P(\lambda) x
$$

and $[P(\lambda)]$ generates the resolution of the identity of $A$ with respect to $\bar{X}_{0}$ (see Remark 6 of $\S 3$ ). Further the set $D(A)$ of all $x$ in $\bar{X}_{0}$ for which the limit in (16) exists is dense in $\bar{X}_{0}$ by Theorem 4 of $\S 3$.

If $A_{0}$ is the infinitesimal generator of $\mathfrak{S}$ with domain $D\left(A_{0}\right)$ then arguing as in [11] it can be shown that $D\left(A_{0}\right)=D(A)$ and $A_{0}=A$. Hence from $\left(16^{\prime}\right)$, the equation (14) of the theorem follows.

This completes the proof of the theorem.

Lemma 6. Let $\mathfrak{S} \equiv[T(\xi): \xi>0]$ be a semi-group of real scalar type operators belonging to a $W^{*}(\|\cdot\|)$-algebra $W$ on $X$. Let $\|T(\xi)\|$ be bounded on every compact subset of $(0, \infty)$. Then $T(\xi)$ is continuous in the strong operator topology for $\xi>0$.

Proof. The argument on p. 591 of [11] in the proof of Lemma 22.3.2 holds here verbatim because of the representation theory in Theorem 7. Also defining $S(\xi)=e^{-\omega_{0} \xi} T(\xi)$ as on p. 591 of [11] we see that $S(\xi)(m)$ is continuous and nonincreasing in $\xi$ for each $m \in \mathfrak{M}$. Thus, as the set of hermitian elements in $\mathfrak{M}$ is a conditionally complete lattice,

$$
\begin{aligned}
\lim _{\xi \rightarrow \xi_{0}^{-}} S(\xi)(m) & =\bigwedge_{\xi<\xi_{0}} S(\xi)(m)=S\left(\xi_{0}\right)(m) \\
& =\bigvee_{\xi>\xi_{0}} S(\xi)(m)=\lim _{\xi \rightarrow \xi_{0}^{+}} S(\xi)(m) .
\end{aligned}
$$

Consequently by Theorem $3, \S 2$, it follows that $\lim _{\xi \rightarrow \hat{\xi}_{0}}-S(\xi) x=$ $\bigwedge_{\xi<\xi_{0}} S(\xi) x=S\left(\xi_{0}\right) x$ and $\lim _{\xi \rightarrow \xi_{0}} S(\xi) x=\bigvee_{\xi>\xi_{0}} S(\xi) x=S\left(\xi_{0}\right) x$. Hence $S(\xi)$ and therefore $T(\xi)$ is strongly continuous at $\xi=\xi_{0}>0$. Hence the lemma.

The above lemma is applied to prove an ergodic theorem for semi-groups of real scalar type operators.

THEOREM 8. Let $\mathfrak{S} \equiv[T(\xi): \xi>0]$ be a semi-group of real scalar type operators of class $X^{*}$ on $X$ and let the members of $\mathfrak{S}$ belong to a $W^{*}(\|\cdot\|)$-algebra $W$. Let $\subseteq$ be of type $\omega_{0}$ and $\omega_{0} \leqq 0$.

Then

$$
\lim _{\xi_{n} \rightarrow \infty} T\left(\xi_{n}\right) x=\left[P(0)-P\left(0^{-}\right)\right] x, x \in X
$$


where $[P(\lambda)]$ generates the resolution of the identity relative to $\bar{X}_{0}$ for the infinitesimal operator $A_{0}$ of $\mathfrak{S}$.

Proof. Since $T(\xi)$ are in the $W^{*}(\|\cdot\|)$-algebra $W\left\|T^{n}(\xi)\right\|=$ $T(\xi) \|^{n}$. Hence arguing as on p. 592 of [11] we have

$$
\|T(\xi)\|=e^{\omega_{0} \xi} \leqq 1
$$

for all $\xi$ in $(0, \infty)$ as $\omega_{0} \leqq 0$. Thus $\|T(\xi)\|$ is uniformly bounded in $(0, \infty)$ and hence by Lemma $6 \subseteq$ is continuous in the strong operator topology, for $\xi>0$. Now as $\subseteq$ satisfies the hypothesis of Theorem 7, making use of the representation given in that theorem we have

$$
T(\xi) x=\int_{-\infty}^{0} e^{\lambda \xi} d P(\lambda) x, x \in X .
$$

Let $P=P(0)-P\left(0^{-}\right)=P\{m: m \in \mathfrak{W}$ and $\alpha(m)=0\}$ in the terminology of the proof of Theorem 7 . Then we have

$$
\begin{aligned}
T(\xi) x-P x & =\int_{\mathfrak{B}} e^{\xi \alpha(m)} d_{m} P(E) x-\int_{\mathfrak{B}_{P}} d_{m} P(E) x \\
& =\int_{-\infty}^{0^{-}} e^{\lambda \xi} d P(\lambda) x \\
& =\lim _{\beta \rightarrow 0^{-}} \int_{-\infty}^{\beta} e^{\lambda \xi} d P(\lambda) x
\end{aligned}
$$

where $\mathfrak{W}_{P}=\{m: m \in \mathfrak{W}$ and $\alpha(m)=0\}$. Now if $f_{n}(\lambda)=e^{\lambda \xi_{n}}$ where $\left\{\xi_{n}\right\}$ is an increasing sequence of positive numbers tending to $\infty$ and $\lambda$ ranges in $(-\infty, \beta), \beta<0$, then $f_{n}(\lambda) \rightarrow 0$ as $\xi_{n} \rightarrow \infty$ for each $\lambda$. Further $f_{1}(\lambda)>f_{n}(\lambda)$. Hence by the generalized Lebesgue bounded convergence theorem on vector valued measures

$$
\lim _{n \rightarrow \infty}\left\|\int_{-\infty}^{\beta} e^{\lambda \hat{\xi} n} d P(\lambda) x\right\|=0
$$

Thus, in view of (17), Lemma 6 of Dunford [8] and Lemma 1 of $\S 2$ we have

$$
\begin{aligned}
& \limsup _{\xi_{n} \rightarrow \infty}\left\|T\left(\xi_{n}\right) x-P x\right\| \\
\leqq & \limsup _{\xi_{n} \rightarrow \infty}\left(\left\|\int_{-\infty}^{\beta} e^{\lambda \hat{\xi} n} d P(\lambda) x\right\|+\left\|\int_{\beta}^{0-} e^{\lambda \xi_{n}} d P(\lambda) x\right\|\right) \\
= & \limsup _{\xi_{n} \rightarrow \infty}\left\|\int_{\beta}^{0-} e^{\lambda \xi_{n}} d P(\lambda) x\right\| \\
\leqq & \limsup _{\xi_{n} \rightarrow \infty} 4 \sup _{\lambda \in[\beta, 0)} e^{\lambda \xi_{n}}\left\|P\left(0^{-}\right) x-P(\beta) x\right\| \\
= & 4\left\|\left(P\left(0^{-}\right)-P(\beta)\right) x\right\| \\
& <\varepsilon
\end{aligned}
$$


if $\beta$ is sufficiently near to zero from below. Hence the theorem.

5. Semi-groups of scalar type operators. In this section we study the spectral representation of semi-groups of scalar type operators when the spectra of the members are not necessarily real.

Definition 10. We shall donote by $\Re_{1}^{4}$ the set $\{R(\lambda, A): \lambda \in \rho(A)\}$ where $A$ is the infinitesimal generator of a semi-group $\subseteq$ and $\rho(A)$ is the resolvent set of $A$.

Lemma 7. If $\mathfrak{S}$ is a semi-group of class $A$ (see p. 321 of [11]) then the commutant of $\mathfrak{S}$ contains the set $\Re_{1}$ and $\mathfrak{S}^{c}=\Re_{1}^{c}$ where $\mathbb{S}^{c}$ and $\Re_{1}^{c}$ are the commutants of $\mathbb{B S}$ and $\Re_{1}$ respectively.

Proof. By Theorem 16.2.1 of [11], $\mathbb{B S}^{c}=\mathfrak{R}_{1}^{c}$. Since $\mathfrak{R}_{1}$ is abelian, $\mathfrak{R}_{1} \subseteq \mathfrak{R}_{1}^{c}=\mathbb{S}^{c}$. Hence the lemma.

Lemma 8. Let $Q(\cdot)$ be an $X^{*}$-countably additive spectral measure over the Borel sets of the complex plane and let $Q(\Delta)=I$ where $\Delta=\left\{\lambda: \operatorname{Re} \lambda \leqq \omega_{0}\right\}$. Suppose $A$ is a closed operator having its spectrum contained in $\Delta$. If $\mu$ is a complex number such that $\operatorname{Re} \mu>\omega_{0}$ and if

$$
R(\mu, A)=\int_{\Delta}(\mu-\lambda)^{-1} d_{\lambda} Q(E)
$$

then

$$
A x=\int_{\Delta} \lambda d_{\lambda} Q(E) x
$$

(the integral in (18) being understood as $\lim _{n \rightarrow \infty} \int_{\sigma_{n}} \lambda d_{\lambda} Q(E) x$ where $\left\{\sigma_{n}\right\}$ is an increasing sequence of Borel sets of $\Delta$ such that $Q\left(\mathbf{U}_{n=1}^{\infty} \sigma_{n}\right)=$ I). Further the integral in (18) exists if and only if $x$ belongs to the domain of $A$. Further $A$ is a spectral operator of scalar type with $Q(\cdot)$ as its resolution of the identity and is unbounded if $\sigma(A)$ is unbounded.

Proof. Since $\operatorname{Re} \mu>\omega_{0}, g(\lambda)=(\mu-\lambda)^{-1}$ is a bounded measurable function defined on the set $\Delta$ and hence by Lemma 6 of Dunford [8]

$$
R(\mu, A)=\int_{\Delta}(\mu-\lambda)^{-1} d_{\lambda} Q(E)
$$

is a bounded scalar type operator of class $X^{*}$ with its resolution of the identity given by

${ }^{4}$ However, in [11] this is denoted by $\Re$. 


$$
Q_{g}(\cdot)=Q\left(g^{-1}(\cdot)\right)
$$

Define $f(\lambda)=\mu-1 / \lambda$. Then let

$$
f(R(\mu, A)) x=\lim _{n \rightarrow \infty} \int_{e_{n}} f(\lambda) d_{\lambda} Q_{g}(E) x
$$

where $e_{n}=\{\lambda:|\lambda| \leqq n$, dist. $(\lambda, 0) \geqq(1 / n)\}$. Then as

$$
Q_{g}(0)=Q\{\lambda: g(\lambda)=0\}=Q(\varphi)=0
$$

the increasing sequence $\left\{e_{n}\right\}$ of bounded Borel sets of the complex plane is such that $Q_{g}\left(\bigcup_{n=1}^{\infty} e_{n}\right)=I$. Hence by Corollary under Theorem 4 of $\S 3$ it follows that $f(R(\mu, A))$ is a spectral operator of scalar type.

Since $f(\lambda)=(\mu-1 / \lambda)$ it is clear that $f(R(\mu, A))=f\left((\mu I-A)^{-1}\right)=A$. Thus

$$
A x=\lim _{n \rightarrow \infty} \int_{e_{n}} f(\lambda) d_{\lambda} Q_{g}(E) x
$$

the limit existing if and only if $x \in D(A)$, where $D(A)$ denotes the domain of $A$.

The resolution of the identity $F(\cdot)$ of $A$ is given by

$$
F(\cdot)=Q_{g}\left(f^{-1}(\cdot)\right)
$$

so that from (19) and (20) it follows that

$$
F(\cdot)=Q(\cdot)
$$

Thus from the relation (21) we have

$$
\begin{aligned}
A x & =\lim _{n \rightarrow \infty} \int_{e_{n}} f(\lambda) d_{\lambda} Q_{g}(E) x \\
& =\lim _{n \rightarrow \infty} \int_{\frac{f\left(e_{n}\right)}{}} \lambda d_{\lambda} Q_{g f}(E) x \\
& =\lim _{n \rightarrow \infty} \int_{\frac{f\left(e_{n}\right)}{}} \lambda d_{\lambda} Q(E) x
\end{aligned}
$$

where $Q_{g f}(\cdot)=Q_{g}\left(f^{-1}(\cdot)\right)$. Now replacing in the above $\overline{f\left(e_{n}\right)}$ by $\overline{f\left(e_{n}\right)} \cap \Delta=\sigma_{n}$, it is easy to check that $\left\{\sigma_{n}\right\}$ is an increasing sequence of bounded Borel sets such that $Q\left(\bigcup_{n=1}^{\infty} \sigma_{n}\right)=I$ and hence

$$
A x=\int_{\Delta} \lambda d_{\lambda} Q(E) x
$$

Since $\sigma(A) \subseteq \Delta, \lambda$ is $Q(\cdot)$-essentially unbounded if $\sigma(A)$ is unbounded and hence in this case $A$ is an unbounded spectral operator of scalar type.

This completes the proof of the lemma. 
Lemma 9. Let $\mathfrak{S} \equiv[S(\xi): \xi>0]$ be a strongly measurable semigroup of scalar type operators of class $X^{*}$ on $X$ and let the members of $\subseteq$ belong to a $W^{*}(\|\cdot\|)$-algebra $W$. Then $\subseteq$ is of finite type $\omega_{0}$ and

$$
\lim _{\xi \rightarrow 0^{+}} S(\xi) x=J x, x \in X
$$

where $J$ is a projection such that $J X=\bar{X}_{0}$ and

$$
J S(\xi)=S(\xi) J=S(\xi)
$$

for all $\tilde{\xi}>0$. If $\bar{X}_{0}=X$ then $\mathfrak{S}$ is $\left(c_{0}\right)$-summable.

Proof. Since $W$ is an abelian $B^{*}$-algebra, and $\subseteq$ is strongly continuous, as it is strongly measurable, the argument on p. 594 of [11] holds here to show that

$$
\|S(\xi)\|=e^{\omega_{0} \xi}
$$

so that except in the trivial case $\widetilde{S} \equiv(0), \omega_{0}$ is finite.

Now let $S^{*}(\xi)$ be the Vidav adjoint of $S(\xi)$ in $W$. Since the *-operation in $W$ is strongly continuous by Theorem 1 of $\S 2, \varsigma^{*} \equiv$ $\left[S^{*}(\xi): \xi>0\right]$ is also a strongly continuous semi-group of scalar type operators of class $X^{*}$.

Let $T(\xi)=S^{*}((\xi / 2)) S((\xi / 2))$. $\quad T(\xi)$ is scalar type of class $X^{*}$ as $T(\xi) \in W$. Since $\|T(\xi)\|$ is uniformly bounded in any finite interval in $(0, \infty)$, from Lemma 6 of $\S 4$ it follows that $T(\xi)$ is also strongly continuous for $\xi>0$. Since $T(\xi)$ belongs to $W$, by Theorem 7 we have a projection $J$ on $X$ such that

$$
J X=\overline{\xi>0} \overline{T(\xi) X}
$$

and

$$
J T(\xi)=T(\xi) J=T(\xi)
$$

for all $\xi>0$. Further $J$ is in $W$.

Now if $\mathfrak{M}$ is the maximal ideal space of $W$, then for $m \in \mathfrak{M}$

$$
|T(\xi)(m)|=S^{*}\left(\frac{\xi}{2}\right)(m) S\left(\frac{\xi}{2}\right)(m)=\left|S\left(\frac{\xi}{2}\right)(m)\right|^{2}
$$

so that

$$
(T(\xi) J)(m)=T(\xi)(m)=\left|S\left(\frac{\xi}{2}\right)(m)\right|^{2} .
$$

From (24) it follows that $S((\xi / 2))(m)=0$ if $J(m)=0$. If $J(m) \neq 0$ then $J(m)=1$ so that 


$$
S\left(\frac{\xi}{2}\right)(m)=S\left(\frac{\xi}{2}\right)(m) J(m)
$$

Hence in all cases we have

$$
(S(\xi) J)(m)=S(\xi)(m)=(J S(\xi))(m)
$$

from which the relation (23) follows.

From (23) we have $\bar{X}_{0} \subseteq \Re(J)$. Since

$$
\begin{gathered}
T(\xi) x=S\left(\frac{\xi}{2}\right) S^{*}\left(\frac{\xi}{2}\right) x \\
\bigcup_{\xi>0} T(\xi) X \cong \bigcup_{\xi>0} S(\xi) X=\bar{X}_{0}
\end{gathered}
$$

and hence we have

$$
\overline{\bigcup_{\xi>0} T(\xi) X}=\Re(J) \subseteq \bar{X}_{0}
$$

Hence $\Re(J)=\bar{X}_{0}$.

The part of the lemma concerning the relation (22) can be proved by arguing as on pp. 317-18 of [11].

When $\bar{X}_{0}=X$ then $J=I$ and hence $\subseteq$ is $\left(c_{0}\right)$-summable.

This concludes the proof of the lemma.

THEOREM 9. Let $\mathfrak{S} \equiv[S(\xi): \xi>0]$ be a strongly measurable semigroup of scalar type operators of class $X^{*}$. Let the members of $\mathfrak{S}$ belong to a $W^{*}(\|\cdot\|)$-algebra $W$. If $\bar{X}_{0}=X$, then $\subseteq$ is $\left(c_{0}\right)$-summable and is of finite type $\omega_{0}$ (say).

Further suppose $\mathfrak{R}_{1} \subseteq W$ (see Definition 7 for $\mathfrak{R}_{1}$ ). Then there exists a unique integral representation of $\subseteq$ in the form

$$
S(\xi) x=\int_{4} e^{\lambda \xi} d_{\lambda} Q(E) x, x \in X
$$

where $Q(\cdot)$ is the resolution of the identity on the Borel sets of the half plane $\Delta=\left\{\lambda: \operatorname{Re} \lambda \leqq \omega_{0}\right\}$ for the infinitesimal generator $A$, where $A$ is given by

$$
A x=\int_{4} \lambda d \lambda_{\lambda} Q(E) x
$$

and the domain $D(A)$ of $A$ is precisely the set of those $x$ in $X$ for which the right hand side of (25) exists. (The integral in (25) is to be understood in the sense given in Lemma 8). Also $A$ is an unbounded spectral operator of scalar type.

Proof. From Lemma 9 it follows that $\subseteq$ is $\left(c_{0}\right)$-summable. Since 
$W$ is a $W^{*}(\|\cdot\|)$-algebra, it is a $B^{*}$-algebra and it contains $\mathfrak{S} \cup \Re_{1}$ by hypothesis. Also the spectrum of any element in $W$ with respect to $\mathfrak{B}(X)$ is the same as that with respect to $W$. Hence by the remark following Theorem 16.2.2 of [11] the algebra $W$ can be substituted for $\mathfrak{B}$ throughout the Chapter XVI of [11]. Arguing in the same way as on p. 595 of [11] and noting that $\|S(\xi)\|=e^{\omega_{0} \xi}$ so that $\omega_{0}$ is finite unless $\subseteq \equiv(0)$ and replacing $\left(P \psi\left(a_{n}\right) x, y\right)$ there by $x^{*} P \psi\left(a_{n}\right) x$ for $x$ in $X$ and $x^{*}$ in $X^{*}$ so that the last line on p.595 of [11] becomes

$$
0=x^{*} P \psi\left(a_{n}\right) x=P^{\prime} x^{*} \psi\left(a_{n}\right) x \rightarrow P^{\prime} x^{*} x
$$

as $n \rightarrow \infty$ (where $P^{\prime}$ is the adjoint operator of $P$ ) and hence $0=x^{*} P x$ for $x \in X$ and $x^{*} \in X^{*}$, we see that the projection $P$ defined on p. 595 of [11] becomes the zero operator. Also arguing exactly in the same way as on p. 596 of [11] we obtain

$$
S(\xi)=\int_{4} e^{\lambda \xi} d \lambda Q(E)
$$

the integral converging in the uniform operator topology and $\Delta=$ $\left\{\lambda: \operatorname{Re} \lambda \leqq \omega_{0}\right\}$.

Now since $\subseteq$ is $\left(c_{0}\right)$-summable, the resolvent of the infinitesimal generator $A$, which is closed, at the point $\mu\left(\operatorname{Re} \mu>\omega_{0}\right)$ is given by

$$
R(\mu, A) x=\int_{0}^{\infty} e^{-\mu \xi} S(\xi) d(\xi) x, x \in X .
$$

Therefore

$$
x^{*} R(\mu, A) x=\int_{0}^{\infty} e^{-\mu \xi}\left[\int_{\Delta} e^{\lambda \xi} x^{*} d_{\lambda} Q(E) x\right] d \xi
$$

and interchanging the order of integration we get

$$
x^{*} R(\mu, A) x=\int_{\Delta}(\mu-\lambda)^{-1} x^{*} d_{\lambda} Q(E) x, x \in X \text { and } x^{*} \in X^{*} .
$$

Since $(\mu-\lambda)^{-1}$ is a bounded Borel measurable function over $\Delta$, $\int_{A}(\mu-\lambda)^{-1} d_{\lambda} Q(E)$ exists in the uniform operator topology and hence from $(26)$ we have

$$
R(\mu, A)=\int_{\Delta}(\mu-\lambda)^{-1} d_{\lambda} Q(E) .
$$

Now all the conditions of Lemma 9 are satisfied by $\mu, \Delta, Q(\cdot)$ and $A$ and hence by Lemma 9 the relation (25) follows, with the domain of $A$ being precisely the set of those $x$ in $X$ for which the right hand side of (25) exists.

Also $\sigma(A)=\alpha(\mathfrak{W})$ (see p. 596 of [11] for the definitions of $\alpha(m)$ 
and $\mathfrak{W}$ ) is unbounded if $\mathfrak{S}$ is strongly continuous and not uniformly continuous. Hence, again by Lemma 9 we conclude that $A$ is an unbounded spectral operator of scalar type with $Q(\cdot)$ as its resolution of the identity.

For the uniqueness of the resolution of the identity of $A$ the same argument in [11] holds here if we replace $(N(\xi) x, N(\eta) y)$ there by $x^{*} S^{*}(\eta) S(\xi) x$ where $S^{*}(\eta)$ is the Vidav adjoint of $S(\eta)$ in $W$ and $x \in X$ and $x^{*} \in X^{*}$.

This completes the proof of the theorem.

6. A generalization of Stone's theorem. In this section we give a generalization of Stone's theorem on one parameter group of unitary operators on Banach spaces, when the group is strongly measurable and its operators belong to a $W^{*}(\|\cdot\|)$-algebra. In [14] we gave the generalized notion of a unitary operator on a Banach space and obtained there an extension of Stone's theorem when the group was uniformly continuous and the underlying Banach space was reflexive.

Definition 11. A spectral operator $U$ of class $X^{*}$ is said to be unitary in the equivalent norm $\|\cdot\|$ of $X$ if $U$ is an onto isometry in the norm $\|\cdot\|$.

Lemma 10. Let $\subseteq \equiv[U(\xi): \xi>0]$ be a strongly measurable semigroup of scalar type operators of class $X^{*}$ and let the members of ऽ belong to a $W^{*}(\|\cdot\|)$-algebra $W$. Let $\Sigma$ be the B.A. of all projections in $W$. Then $U(\xi)$ are invariant on $\mathfrak{M}(x)$ for each $x \in X$ where $\mathfrak{M}(x)$ is the closed subspace spanned by the set $\{E x: E \in \Sigma\}$. If the restriction of $U(\xi)$ on $\mathfrak{M}(x)$ be denoted by $U_{x}(\xi)$ then $\Im_{x} \equiv\left[U_{x}(\xi): \xi>0\right]$ is again a semi-group of scalar type operators of class $\{\mathfrak{M}(x)\}^{*}$ on $\mathfrak{M}(x)$ and is strongly continuous on $\mathfrak{M}(x)$. If $A_{x}$ is the infinitesimal generator of $\mathfrak{S}_{x}$ and $A$ is that of $\mathfrak{S}$ then

$$
A / \mathfrak{M}(x)=A_{x} .
$$

Proof. If $E_{\xi}(\cdot)$ is the resolution of the identity of $U(\xi)$ then it is easy to verify that $U(\xi)$ is invariant on $\mathfrak{M}(x)$ and $E_{\xi x}(\cdot)=E_{\xi}(\cdot) / \mathfrak{M}(x)$ is the resolution of the identity of the restriction $U_{x}(\xi)$ on $\mathfrak{M}(x)$. Also $U_{x}(\xi)$ is a scalar type operator on $\mathfrak{M}(x)$ of class $\{\mathfrak{M}(x)\}^{*}$ as the set function $E_{\xi x}(\cdot) y, y \in \mathfrak{M}(x)$ is countably additive. The strong continuity of $\mathfrak{S}_{x}$ is a consequence of that of $\mathfrak{S}$. It is easy to establish that $A_{x}$ is the restriction of $A$ to $\mathfrak{M}(x)$. Hence the lemma.

Theorem 10. (A generalization of Stone's theorem on one-parameter group of unitary operators to Banach spaces). Let 


$$
\subseteq \equiv[U(\xi):-\infty<\xi<\infty]
$$

be a group of scalar type operators of class $X^{*}$ on $X$. Let $U(\xi)$ belong to a $W^{*}(\|\cdot\|)$-algebra $W$ and let their spectrum lie on the unit circle. Further let $\subseteq$ be strongly measurable.

Then $\mathfrak{S}$ is continuous in the strong operator topology and $U(\xi)$ are unitary in the norm $\|\cdot\|$. Further there exists a unique representation for $U(\xi)$ of the form

$$
U(\xi) x=\int_{-\infty}^{\infty} e^{i \lambda \xi} d_{\lambda} Q^{\prime}(E) x, x \in X
$$

with $Q^{\prime}(E)=Q\{\lambda: i \lambda \in E\}$ for Borel sets $E$ of the complex plane where $Q(\cdot)$ is the resolution of the identity for the infinitesimal generator $A$ given by

$$
A x=i \int_{-\infty}^{\infty} \lambda d_{\lambda} Q^{\prime}(E) x
$$

and the domain $D(A)$ of $A$ is precisely the set of all $x$ in $X$ for which the integral in (27) exists.

Proof. That $U(\xi)$ is strongly continuous follows from the hypothesis that $U(\xi)$ is strongly measurable. Since $U(\xi)$ belongs to the $W^{*}(\|\cdot\|)$-algebra $W$ and since the spectrum of $U(\xi)$ lies on the unit circle it is easy to show that $U(\xi)=e^{i R_{\xi}}$ for some operator $R_{\xi}$ in $W$, which is hermitian in the norm $\|\cdot\|$. Hence $\|U(\xi)\|=\left\|e^{i R_{\xi}}\right\|=1$ for $-\infty<\xi<\infty$ by Lemma 1 of Vidav [18]. Also

$$
\left\|U^{-1}(\xi)\right\|=\|U(-\xi)\|=1
$$

and hence $U(\xi)$ are onto isometry in $\|\cdot\|$. But, as $U(\xi)$ are scalar type $U(\xi)$ are unitary in $\|\cdot\|$.

All the conditions of Theorem 9 will be satisfied by $\subseteq$ if we show that $\Re_{1} \subseteq W$. In view of Theorems 4.3 and 4.5 of Bade [3] to prove $\Re_{1} \subseteq W$ it suffices to show that the resolvent operator $R(\lambda, A)$ of the infinitesimal generator $A$ leaves invariant each $\mathfrak{M}(x)$ for $x \in X$, where $\mathfrak{M}(x)$ is the closed subspace spanned by $\{E x: E \in \Sigma\}, \Sigma$ being the B.A. of all projections in $W$. Now to prove this, let $U_{x}(\xi)$ be the restriction of $U(\xi)$ on $\mathfrak{M}(x)$. Then as said in the proof of Lemma $10, U_{x}(\xi)$ are scalar type operators on $\mathfrak{M}(x)$, with their resolutions of the identity being given by $E_{\xi x}(\cdot)=E_{\xi}(\cdot) / \mathfrak{M}(x)$. Hence it will follow that $U_{x}(\xi)$ are onto isometries of $\mathfrak{M}(x)$ in the norm $\|\cdot\|$. Therefore, the set $\Lambda=\{\lambda: \operatorname{Re} \lambda \neq 0\}$ is dense in the resolvent set $\rho\left(A_{x}\right)$ of the infinitesimal generator $A_{x}$ of $\mathfrak{S}_{x} \equiv\left[U_{x}(\xi):-\infty<\xi<\infty\right]$. Thus for a $\lambda \in \Lambda,\left(\lambda I-A_{x}\right)^{-1}$ is an everywhere defined operator on $\mathfrak{M}(x)$, as $A_{x}$ is closed. As $\subseteq$ is a group of isometries on $X, \Lambda$ is also dense in the resolvent set $\rho(A)$ 
and hence for $\lambda \in \Lambda,(\lambda I-A)^{-1}$ exists as an everywhere defined operator on $X$ and is an extension of $\left(\lambda I-A_{x}\right)^{-1}$ because of Lemma 10. Thus $(\lambda I-A)^{-1}=\left(\lambda I-A_{x}\right)^{-1}$ on $\mathfrak{M}(x)$. But $\left(\lambda I-A_{x}\right)^{-1} \mathfrak{M}(x) \subseteq \mathfrak{M}(x)$ so that $(\lambda I-A)^{-1} \mathfrak{M}(x) \subseteq \mathfrak{M}(x)$. Since $\Lambda$ is dense in $\rho(A)$, it follows that $R(\lambda, A) \mathfrak{M}(x) \subseteq \mathfrak{M}(x)$ for all $\lambda \in \rho(A)$.

Thus the semi-group $\mathfrak{S}^{1} \equiv[U(\xi): \xi>0]$ satisfies the hypothesis of Theorem 9 and hence the proof of Theorem 9 holds verbatim. As $U(\xi)$ are in $W$ with their spectrum on the unit circle, it follows that the range of $U(\xi)(m)$ is the unit circle so that $|U(\xi)(m)|=1$ for $m \in \mathfrak{M}$, where $\mathfrak{M}$ is the maximal ideal space of $W$. Thus the function $\alpha(m)$ defined in the proof of Theorem 9 is purely imaginary and $\alpha(\mathfrak{W})=\sigma(A)$ reduces to a subset of the imaginary axis. Hence we have

$$
\begin{aligned}
U(\xi) x & =\int_{4} e^{\lambda \xi} d_{\lambda} Q(E) x \\
& =\int_{-\infty}^{\infty} e^{i \lambda \xi} d_{\lambda} Q^{\prime}(E) x
\end{aligned}
$$

where $Q^{\prime}(E)=Q[\lambda: i \lambda \in E]$ and

$$
\begin{aligned}
A x & =\int_{\Delta} \lambda^{\prime} d_{\lambda^{\prime}} Q(E) x \\
& =i \int_{-\infty}^{\infty} \lambda d_{\lambda} Q^{\prime}(E) x .
\end{aligned}
$$

Further the domain of $A$ is precisely the set of those $x$ in $X$ for which the integral in (27) exists. Also $Q(\cdot)$ is the resolution of the identity of $A$.

For $\xi<0$,

$$
\begin{aligned}
U(\xi) & =U^{*}(-\xi)(\text { Vidav adjoint of } U(-\xi)) \\
& =\left[\int_{-\infty}^{\infty} e^{-i \lambda \xi} d \lambda Q^{\prime}(E)\right]^{*} \\
& =\int_{-\infty}^{\infty} e^{i \lambda \xi} d_{\lambda} Q^{\prime}(E)
\end{aligned}
$$

as the spectral projections $Q^{\prime}(E)$ are in $W$ and hence are hermitian in $\|\cdot\|$ by Remark 5 of $\S 2$. For $\xi=0$ the representation clearly holds.

This completes the proof of the theorem.

REMARK 7. In all the above theorems on semi-groups of operators, the hypothesis that the members of the semi-group belong to a $W^{*}(\|\cdot\|)$-algebra can be replaced by an equivalent hypothesis that the B.A. $\Sigma$ determined by the resolutions of the identity of the members of the semi-group is $\sigma$-complete. When the Banach space $X$ is weakly complete, it suffices to assume that $\Sigma$ is bounded. Here in Theorem 10 we have proved the extension of Stone's theorem to arbitrary Banach 
spaces. On the other hand in [6] Berkson has obtained the extension but for weakly complete Banach spaces with a weaker hypothesis that there is a uniform bound on the resolutions of the identity of all operators to $U(\xi)$. The fact that these different resolutions of the identity generate a bounded B.A. is obtained as a corollary of his method of proof.

The author wishes to express his deep sense of gratitude to professor V. K. Balachandran, under whose guidance the thesis was written. The author also wishes to express his heart-felt thanks to Professor W.G. Bade and Professor Ralph S. Phillips for their helpful comments and suggestions.

\section{REFERENCES}

1. W. G. Bade, Unbounded spectral operators, Pacific J. Math. 4 (1954), 373-392.

2. Weak and strong limits of spectral operators, Pacific J. Math. 4 (1954), $393-413$.

3. On Boolean algebras of projections and algebras of operators, Trans. Amer. Math. Soc. 80 (1955), 345-360.

4. E. Berkson, A characterisation of scalar type operators on reflexive Banach spaces, Pacific J. Math. 13 (1963), 365-373.

5. — Some characterisations of $C^{*}$-algebras, Illinois J. Math. 10 (1966), 1-8.

6. Semi-groups of scalar type operators and a theorem of Stone, Illinois J. Math. 10 (1966), 345-352.

7. J. Dieudonné, Sur la bicommutante d'une algèbra d'opérateurs, Portugaliae Math. 14 (1955), 35-38.

8. N. Dunford, Spectral operators, Pacific J. Math. 4 (1954), 321-354.

9. D. A. Edwards and C. T. Ionescu Tulcea, Some remarks on commutative algebras of operators on Banach spaces, Trans. Amer. Math. Soc. 93 (1959), 541-551.

10. S. R. Foguel, The relation between a spectral operator and its scalar part, Pacific J. Math. 8 (1958), 51-65.

11. E. Hille and R. S. Phillips, Functional analysis and semi-groups, Amer. Math. Soc. Colloquium Publications 31, 1957.

12. G. Lumer, Semi-inner-product spaces, Trans. Amer. Math. Soc. 100 (1961), 29-43. 13. Spectral operators, hermitian operators and bounded groups, Acta. Sci. Math. (Szeged) 25 (1964), 75-85.

14. T. V. Panchapagesan, Unitary operators in Banach spaces, Pacific J. Math. 22 (1967), 465-475.

15. R. S. Phillips, Spectral theory for semi-groups of linear operators, Trans. Amer. Math. Soc. 71 (1951), 393-415.

16. C. E. Rickart, General theory of Banach algebras, D. Van Nostrand, New York, 1960.

17. M. H. Stone, Linear transformations in Hilbert space and their applications to analysis, Amer. Math. Soc. Colloquium Publications 15, 1951.

18. I. Vidav, Eine metrische Kennzeichnung der selbst adjungierten Operatoren, Math. Z. 66 (1956), 121-128.

Received March 1, 1968. This paper forms a part of the author's Ph. D. dissertation (October 1965) of the University of Madras. 



\title{
PACIFIC JOURNAL OF MATHEMATICS
}

\author{
EDITORS
}

H. ROYDEN

Stanford University

Stanford, California

R. R. PhelPS

University of Washington

Seattle, Washington 98105
J. DUGUNDJI

Department of Mathematics

University of Southern California

Los Angeles, California 90007

RICHARD ARENS

University of California

Los Angeles, California 90024

\section{ASSOCIATE EDITORS}
E. F. BECKENBACH
B. H. NeumanN
F. WOLF
K. YoSHIDA

\section{SUPPORTING INSTITUTIONS}

\author{
UNIVERSITY OF BRITISH COLUMBIA \\ CALIFORNIA INSTITUTE OF TECHNOLOGY \\ UNIVERSITY OF CALIFORNIA \\ MONTANA STATE UNIVERSITY \\ UNIVERSITY OF NEVADA \\ NEW MEXICO STATE UNIVERSITY \\ OREGON STATE UNIVERSITY \\ UNIVERSITY OF OREGON \\ OSAKA UNIVERSITY \\ UNIVERSITY OF SOUTHERN CALIFORNIA
}

\author{
STANFORD UNIVERSITY \\ UNIVERSITY OF TOKYO \\ UNIVERSITY OF UTAH \\ WASHINGTON STATE UNIVERSITY \\ UNIVERSITY OF WASHINGTON \\ $\stackrel{*}{*} \stackrel{*}{*}{ }^{*}{ }^{*}$ \\ CHEVRON RESEARCH CORPORATION \\ TRW SYSTEMS \\ NAVAL WEAPONS CENTER
}

The Supporting Institutions listed above contribute to the cost of publication of this Journal, but they are not owners or publishers and have no responsibility for its content or policies.

Mathematical papers intended for publication in the Pacific Journal of Mathematics should be in typed form or offset-reproduced, double spaced with large margins. Underline Greek letters in red, German in green, and script in blue. The first paragraph or two must be capable of being used separately as a synopsis of the entire paper. It should not contain references to the bibliography. Manuscripts, in duplicate if possible, may be sent to any one of the four editors. Please classify according to the scheme of Math. Rev. 36, 1539-1546. All other communications to the editors should be addressed to the managing editor, Richard Arens, University of California, Los Angeles, California, 90024.

50 reprints are provided free for each article; additional copies may be obtained at cost in multiples of 50 .

The Pacific Journal of Mathematics is published monthly. Effective with Volume 16 the price per volume (3 numbers) is $\$ 8.00$; single issues, $\$ 3.00$. Special price for current issues to individual faculty members of supporting institutions and to individual members of the American Mathematical Society: $\$ 4.00$ per volume; single issues $\$ 1.50$. Back numbers are available.

Subscriptions, orders for back numbers, and changes of address should be sent to Pacific Journal of Mathematics, 103 Highland Boulevard, Berkeley, California, 94708.

PUBLISHED BY PACIFIC JOURNAL OF MATHEMATICS, A NON-PROFIT CORPORATION

Printed at Kokusai Bunken Insatsusha (International Academic Printing Co., Ltd.), 7-17, Fujimi 2-chome, Chiyoda-ku, Tokyo, Japan. 


\section{Pacific Journal of Mathematics \\ Vol. 30, No. $2 \quad$ October, 1969}

Gregory Frank Bachelis, Homomorphisms of annihilator Banach algebras.

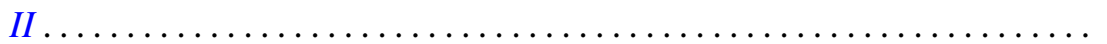

Leon Bernstein and Helmut Hasse, An explicit formula for the units of an algebraic number field of degree $n \geq 2 \ldots \ldots \ldots \ldots \ldots \ldots \ldots . \ldots 29$

David W. Boyd, Best constants in a class of integral inequalities ........ 367

Paul F. Conrad and John Dauns, An embedding theorem for lattice-ordered

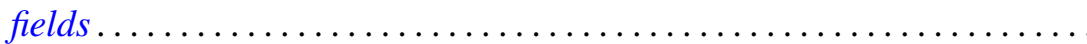

H. P. Dikshit, Summability of Fourier series by triangular matrix

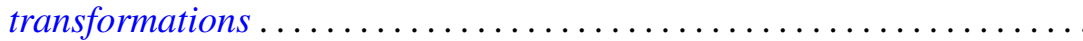

Dragomir Z. Djokovic, Linear transformations of tensor products preserving a fixed rank............................. 411

John J. F. Fournier, Extensions of a Fourier multiplier theorem of Paley . . . 415 Robert Paul Kopp, A subcollection of algebras in a collection of Banach

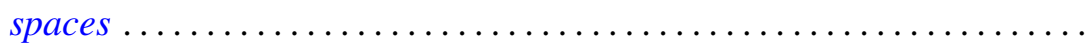

Lawrence Louis Larmore, Twisted cohomology and enumeration of vector bundles ...................................... 437

William Grenfell Leavitt and Yu-Lee Lee, A radical coinciding with the lower radical in associative and alternative rings .................

Samuel Merrill and Nand Lal, Characterization of certain invariant subspaces of $H^{p}$ and $L^{p}$ spaces derived from logmodular

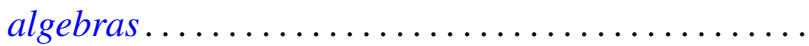

Sam Bernard Nadler, Jr., Multi-valued contraction mappings ....

T. V. Panchapagesan, Semi-groups of scalar type operators in Banach spaces ....................................

J. W. Spellmann, Concerning the infinite differentiability of semigroup motions

H. M. (Hari Mohan) Srivastava, A note on certain dual series equations involving Laguerre polynomials.

Ernest Lester Stitzinger, A nonimbedding theorem of associative algebras................................

J. Jerry Uhl, Jr., Martingales of vector valued set functions ...

John Mays Worrell Jr., On continuous mappings of metacompact $\check{C} e c h$

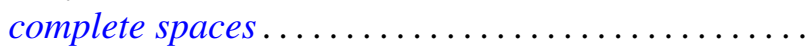

